\title{
Asymptomatic ST segment elevation in the recovery phase of the exercise stress test due to slow coronary flow
}

\author{
Murat Sunbul, Okan Erdogan, Ibrahim Sari \\ Department of Cardiology, School of Medicine, Marmara University, Istanbul, Turkey
}

Postep Kardiol Inter 2014; 10, 1 (35): 53-56 DOI: $10.5114 /$ pwki.2014.41471

\begin{abstract}
A bstract
Exercise-induced ST segment elevation without Q wave formation is rarely observed. Frequent causes are significant coronary stenosis, myocardial bridge and coronary vasospasm. Both exercise-induced ST segment elevation due to slow coronary flow and ST segment elevation in the recovery phase of the exercise stress test are very rare. We present a 49-year-old man with asymptomatic inferolateral ST segment elevation in the recovery phase of the exercise stress test due to slow coronary flow, which has not been reported previously. The learning points of the present paper are as follows: a) although significant coronary stenosis, myocardial bridge and coronary vasospasm are frequent causes of exercise-induced ST segment elevation, slow coronary flow might also cause it; b) one should keep in mind that ST segment elevation might also occur in the recovery phase; and c) ST segment elevation might be asymptomatic, as in the present case.
\end{abstract}

Key words: coronary slow flow, ST segment, exercise test, recovery, ischemia.

\section{Introduction}

Exercise-induced ST segment elevation without Q wave formation is a rare situation. Frequent causes are significant coronary stenosis, myocardial bridge and coronary vasospasm [1-4]. Slow coronary flow is an angiographic finding defined as the slow movement of contrast throughout the coronary lumen in the absence of epicardial coronary stenosis. Exercise-induced ST segment elevation is a very rare situation for patients with slow coronary flow.

We present here a rare case of asymptomatic inferolateral ST segment elevation in the recovery phase of the exercise stress test due to slow coronary flow, which has not been reported previously.

\section{Case report}

A 49-year-old man presented with stable angina for more than 6 months. He had a history of smoking and hyperlipidemia. On admission physical examination, resting electrocardiogram and transthoracic echocardiography were normal. He underwent a treadmill exercise test with the Bruce protocol. The patient tolerated the exercise stress test (he was able to exercise for 9 min and the maximal heart rate was $158 / \mathrm{min})$. However, during the recovery period ( $2 \mathrm{~min} 57 \mathrm{~s}$ after starting the recovery period) he suddenly developed ST segment elevation in leads D2, D3, aVF and V4-6 associated with reciprocal ST segment depression in leads D1, aVL and V1-V3 (Figure 1). The patient was asymptomatic. After sublingual nitroglycerin administration ST segment elevation gradually resolved in about $30 \mathrm{~min}$. Coronary angiography (within 45 min of diagnosis) revealed normal coronary arteries with no apparent stenosis. However, coronary flow was significantly slowed in all coronary arteries (Figure 2). The thrombolysis in myocardial infarction (TIMI) frame count was 68 for the left anterior descending artery (normal: $36 \pm 1$ ), 52 for the circumflex artery (normal: $22.2 \pm 4$ ) and 44 for the right coronary artery (normal: $20.4 \pm 3$ ). We planned to perform a provocative test; however, at that time neither ergonovine nor acetylcholine was present in our cath lab. Instead, we performed a hyperventilation test during coronary angiography, which was negative. The patient was reassured and discharged with acetylsalicylic acid $100 \mathrm{mg}$ q.d., diltiazem $90 \mathrm{mg}$ b.i.d., atorvastatin $20 \mathrm{mg}$ q.d. and isosorbide mononitrate $40 \mathrm{mg}$ b.i.d.

\section{Corresponding author:}

Murat Sunbul MD, Marmara University Education and Research Hospital, Pendik, Istanbul, Turkey, phone: +90 5065819015 , fax: +90 21665706 95, e-mail: drsunbul@yahoo.com.tr

Received: 1.09.2013, accepted: 9.01.2014. 


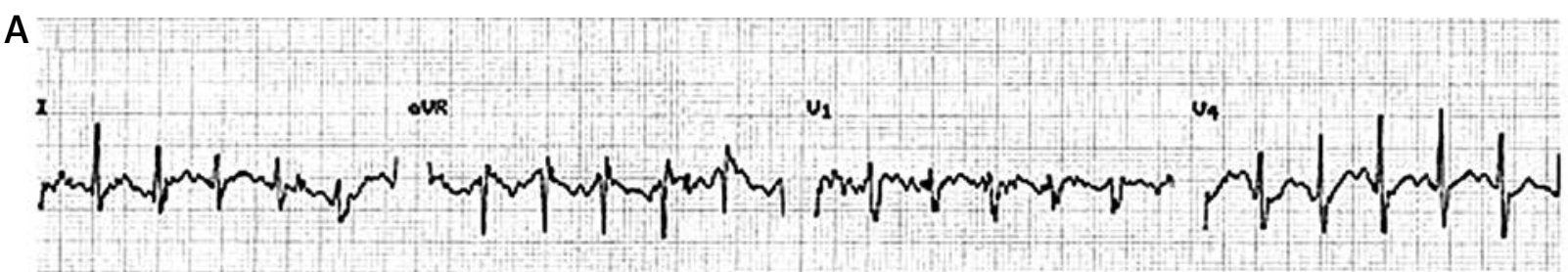

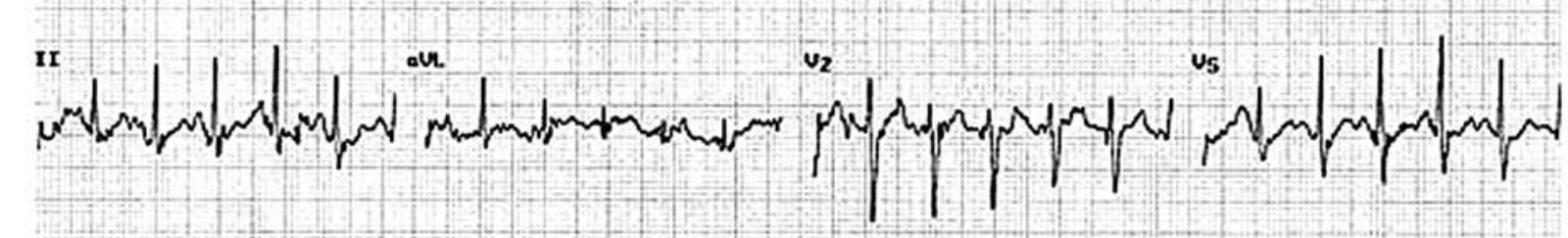

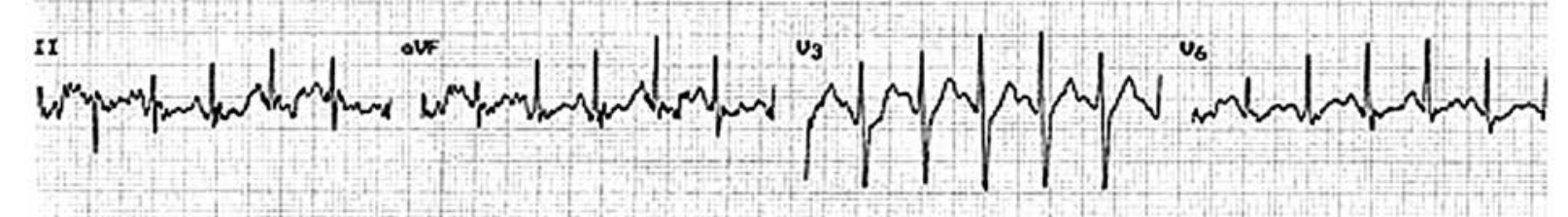

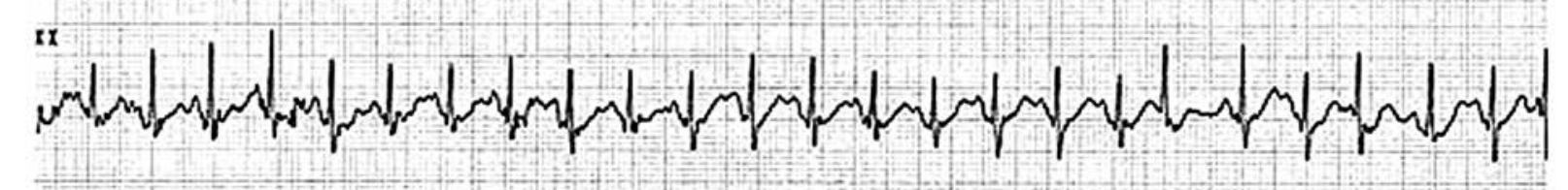

${ }_{3}$

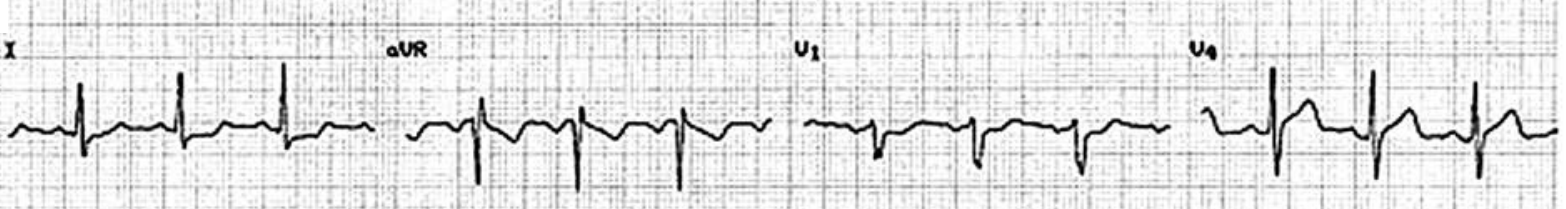

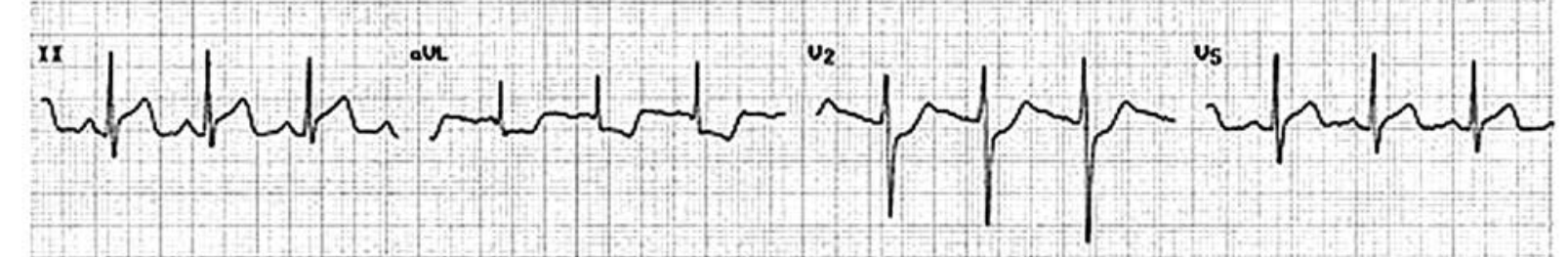

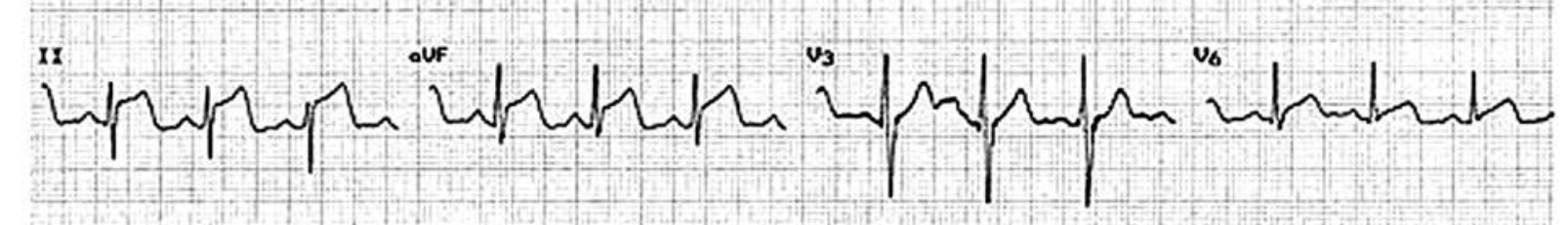

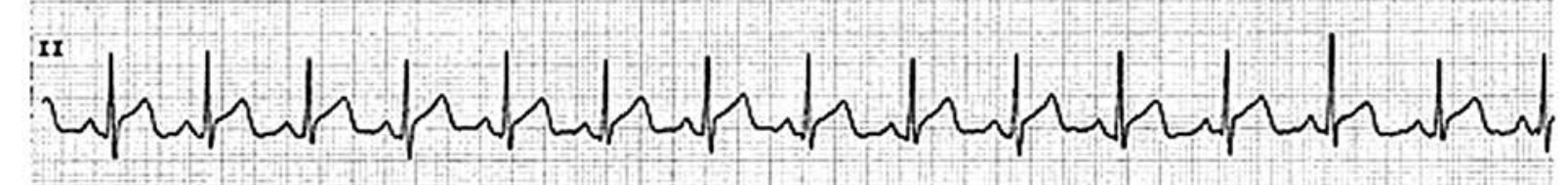



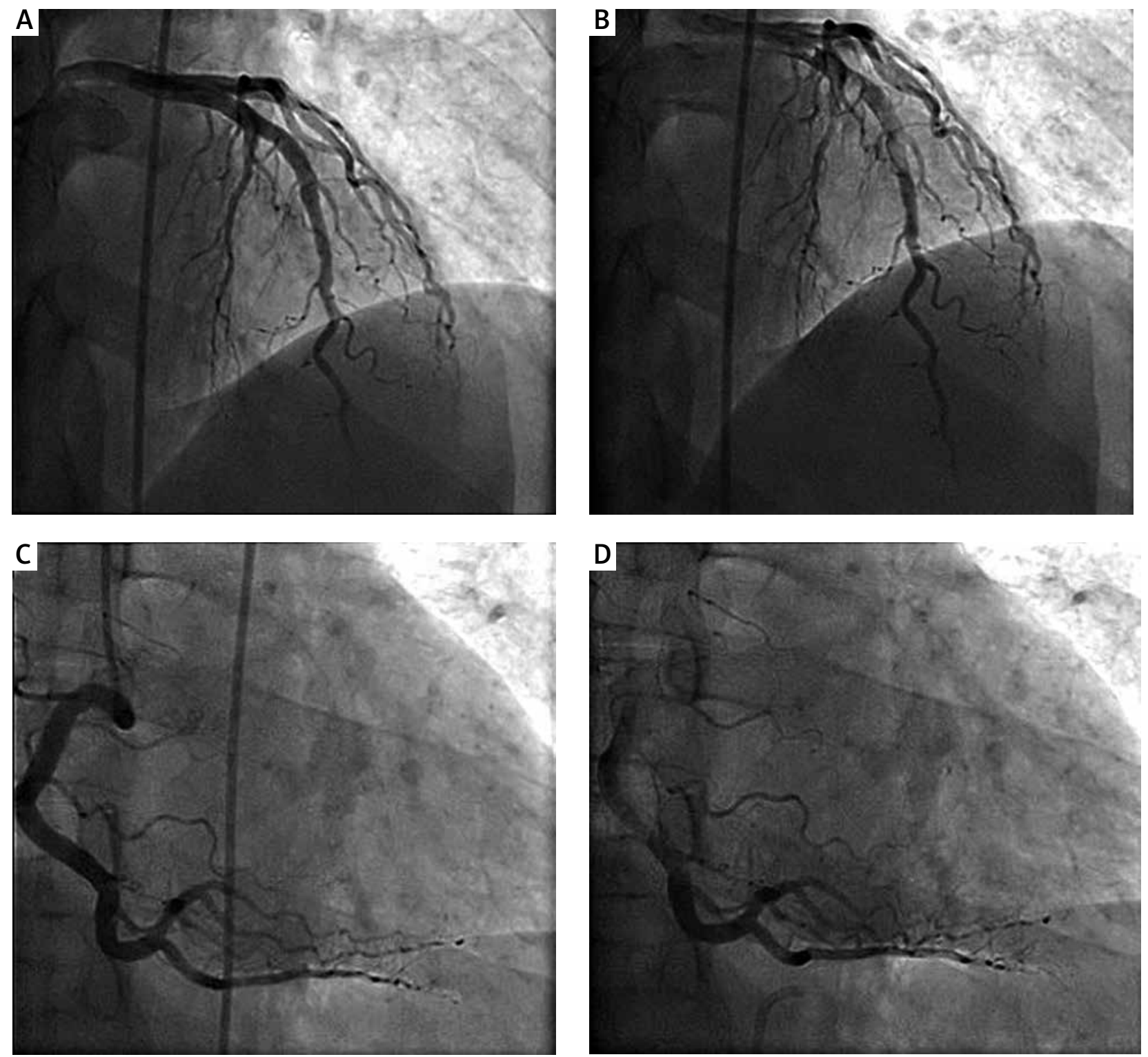

Figure 2. Right cranial view of left anterior descending and circumflex arteries during filling (A) and wash-out phase (B). Right anterior oblique view of right coronary artery during filling (C) and wash-out phase (D)

\section{Discussion}

In the present paper, we report a 49-year-old man with asymptomatic inferolateral ST segment elevation in the recovery phase of the exercise stress test due to slow coronary flow, which has not been reported previously.

Exercise-induced ST segment elevation without Q wave formation is rarely observed. Its frequent causes are significant coronary artery stenosis, myocardial bridge and coronary vasospasm [1-4]. Exercise-induced ST segment elevation is a very rare situation for patients with slow coronary flow. To date, there is only one paper reporting exercise-induced ST segment elevation due to slow coronary flow [5]. One of the interesting points of the present case is the occurrence of ST segment ele- vation in the recovery phase of exercise stress, which is even more rare [6].

One possible etiologic explanation for the present case is coronary vasospasm. However, clinical and angiographic findings were not compatible with variant angina. The patient did not describe any anginal episodes at rest, especially during the morning. Additionally, the coronary angiogram of our patient was angiographically normal without any apparent stenosis. In the majority of patients with coronary vasospasm, significant eccentric proximal coronary obstruction of at least one coronary artery has been reported [5]. For these reasons we considered that ST elevation occurred due to slow coronary flow. 


\section{Conclusions}

The learning points of the present paper are as follows: a) although significant coronary stenosis, myocardial bridge and coronary vasospasm are frequent causes of exercise-induced ST segment elevation, slow coronary flow might also cause it; b) one should keep in mind that ST segment elevation might also occur in the recovery phase; and c) ST segment elevation might be asymptomatic, as in the present case.

\section{References}

1. Ozmen N, Yiginer O, Uz O, et al. ST segment elevation in the lead aVR during exercise treadmill testing may indicate left main coronary artery disease. Kardiol Pol 2010; 68: 1107-1111.

2. Abdou M. Myocardial bridging causing ischemia and recurrent chest pain: a case report. Int Arch Med 2011; 7: 24.

3. Inomata T, Igarashi Y, Ebe K, et al. Exercise-induced angina with intermittent ST-segment elevation. Inter Med 1995; 34: 597-601.

4. Kapoor A, Goel PK, Gupta S. Slow coronary flow - a cause for angina with ST segment elevation and normal coronary arteries. A case report. Int J Cardiol 1998; 67: 257-261.

5. Celik T, Iyisoy A, Kursaklioglu H, et al. ST elevation during treadmill exercise test in a young patient with slow coronary flow: a case report and review of literature. Int J Cardiol 2006; 112: e1-e4.

6. Piszczek S, Dziuk M, Mazurek A, et al. ST-segment elevation in the recovery phase of nuclear exercise stress test with $99 \mathrm{mTc}$-sestamibi in a patient with critical RCA stenosis and subtle systolic dysfunction in speckle tracking imaging. Nucl Med Rev 2012; 1: 75-79. 